\title{
Modeling Compression-After-Impact Response of Polymer Matrix Composites Subjected to Seawater Aging
}

\author{
Arun Krishnan, Caglar Oskay* \\ Department of Civil and Environmental Engineering \\ Vanderbilt University \\ Nashville, TN, 37235
}

\begin{abstract}
This manuscript presents a computational investigation of the compression-after-impact (CAI) response of polymer matrix composites subjected to seawater induced environmental degradation. A multiscale computational model that accounts for seawater-induced property degradation in composite constituents is proposed to predict the CAI response of E-glass reinforced vinyl-ester composites. Predictions of the CAI response as a function of specimen saturation are validated against experimental observations. The investigations revealed that partially saturated vinyl-ester matrix composites undergo a significant reduction in the CAI strength, part of which is recovered upon full matrix saturation. The proposed computational model captures the response characteristics of partially- and fully-saturated specimens.
\end{abstract}

Keywords: Polymer-matrix composites (PMCs), Environmental Degradation, Matrix cracking, Damage Mechanics, Multiscale modeling, Compression-after-impact

\section{Introduction}

Fiber-reinforced polymer composites provide tremendous potential as structural components in naval and marine structures due to their high specific stiffness, strength and durability properties. For instance, polymer composites with glass reinforcements (GFRP) are increasingly used in marine structures including radomes, submarine casings, sonar casings, propellers, masts and shafts [1] as well as light-weight advanced defense platform structures due to high mobility and low fuel cost replacements [2]. Vinyl-ester and polyesters are

\footnotetext{
${ }^{*}$ Corresponding Author: VU Station B\#351831, 2301 Vanderbilt Place, Nashville, TN 37235. Email: caglar.oskay@vanderbilt.edu
} 
preferred over epoxy as matrix materials due to their low cost and ease of processing [2]. E-Glass fibers are typically preferred compared to carbon fibers due to the low cost and higher achievable ultimate strain levels, despite their reduced tolerance to aqueous environments in comparison to carbon fibers [2].

Environmental degradation of the mechanical properties including strength, stiffness, compression-after-impact, and immersed fatigue strength of fiber reinforced polymer composites subjected to seawater environment is of significant concern. Prior experimental investigations $[3,4]$ focused on characterization of composite specimens fully immersed in seawater. Liao et al. [5] show a reduction in strength and moduli of pultruded GFRP composites with environmental aging in salt solutions, and suggest several mechanisms for flexural strength degradation including significant degradation in the fiber-matrix interphase region and in-situ fiber strength degradation. Karbhari and Zhang [2] demonstrate reduction in tensile strength of E-Glass vinyl-ester (EVE) composites. They suggest that the changes in strength are not only due to plasticization and hydrolysis of the matrix, but also due to degradation at the fiber-matrix interface and at the individual fiber itself. Roy et al. [6] report a decrease in flexural strength of glass fiber reinforced vinyl-ester composites of approximately $17 \%$ when subjected to boiling water.

Compression-after-impact (CAI) strength of composite structures exposed to seawater was shown to be lower than dry structures. Imielinska and Guillaumat [3] show that the CAI strength of woven aramid-glass fiber epoxy composites reduced by a maximum of $42 \%$ under low velocity impact. In view of the sensitivity of the CAI response to damage distribution within composite specimens, $\mathrm{Xu}$ et al.. [7] experimentally investigated the CAI response of composite specimens exposed to seawater from one side only, in contrast to full immersion of specimens in seawater. One side only exposure was argued to describe the seawater exposure more accurately for marine structures.

In this manuscript, the effect of seawater exposure on the compression-after-impact strength of polymer composites is numerically investigated. A multiscale computational model that accounts for seawater-induced degradation of composite constituents is devised to numerically study the evolution of CAI strength as a function of seawater ingress into the 
composite. The proposed model is employed to predict the CAI of a suite of vinyl-ester specimens subjected to seawater for up to 30 months in an experimental study. The investigations reveal that partially saturated composites undergo a significant reduction of CAI strength, part of which is recovered upon saturation. The proposed seawater degradation model captures the response characteristics of partially- and fully-saturated composites.

The remainder of this manuscript is organized as follows: A summary of experiments employed to validate the proposed computational environmental degradation model is described in Section 2. Section 3 describes the computational multiscale approach to model the response of composites including seawater ingress induced degradation of material properties. Moisture absorption model and degradation of constituent material properties as a function of saturation are described. In Section 4, numerical investigations of the CAI response of partially saturated and fully saturated E-glass vinyl-ester composite specimens is presented. Conclusions and future research directions are described in Section 5.

\section{Experimental Investigations}

A suite of experiments were conducted to characterize the effect of seawater exposure on the CAI strength of EVE composites [7, 8]. Composite specimens were manufactured using vaccum assisted resin transfer molding process [9]. Eight plain weave fabric layers were stitched to produce rectangular composite panels with dimensions of $101.6 \pm 0.1 \mathrm{~mm} x$ $152.4 \pm 0.1 \mathrm{~mm}(4 " \times 6 " \pm 0.004)$. The thickness of each panel is $4.96 \mathrm{~mm} \pm 0.22 \mathrm{~mm}$ which is required by ASTM D 7137. The samples were exposed to sea water on one side only, (Fig. 1a) to mimic realistic exposure conditions observed in naval structures. This is in contrast with previous approaches, where the experimental samples are fully immersed in water. Artificial seawater with a salinity content of approximately 2.9\% (ASTM D1141) is used as the moisture environment. Composite specimens with varying degrees of exposure (i.e, approximately 3 month intervals of up to 30 months) were prepared for CAI testing.

CAI testing consists of subjecting the moisture exposed composite specimens to impact damage followed by compression testing as illustrated in Fig. 1b-c. In the impact testing, a $16 \mathrm{~mm}\left(5 / 8^{\prime \prime}\right)$ diameter hemispherical impactor impinges on the composite specimen with 60 
Joules of energy using a drop tower apparatus as prescribed by ASTM D 7137. The impact damage causes a horizontal and a vertical matrix crack on the front-impact surface (Fig. 1d) while delamination zones along with fiber breakage was observed on the back surface.

Delamination zones are approximate circles with increasing radii towards the back face with maximum radius of about $17.6 \pm 1 \mathrm{~mm}$ observed between the last two plies. No significant change in the delamination zone is observed in the specimens as a function of moisture exposure duration. Fiber breakage also occurred at the drop weight impact site.

The impacted samples were then tested in compression using a compression-after-impact fixture prescribed by the ASTM D 7137. Under a loading rate of 1 $\mathrm{mm} / \mathrm{min}$, the impacted samples were tested under uniaxial compression loading until failure. The delamination zone from the impact damage extends along the horizontal direction and the final failure is controlled by sublaminate buckling. While the horizontal matrix crack grows laterally across the specimen, the vertical matrix crack does not increase in length under compression loading as shown in Fig. 1e. A shear crack at an angle of approximately $30^{\circ}-45^{\circ}$ was observed along the thickness of the specimens. This failure pattern is consistently observed in all tested specimens (Fig. 1f). Table 1 summarizes the CAI strength of moisture-exposed specimens. The slight rise in the CAI specimens subjected to 4-months of moisture exposure is attributed to the larger specimen thickness. Closer examination with an SEM indicates greater fiber exposure to seawater in case of wet specimens in comparison with dry specimens as reported in Ref. [7].

\section{Moisture Absorption Model}

Moisture absorption process in EVE and other polymer matrix composites is typically idealized using Fick's second law of diffusion:

$$
\frac{\partial \bar{s}}{\partial t}=\bar{D} \frac{\partial^{2} \bar{s}}{\partial z^{2}}
$$

in which, $\bar{s}$ denotes degree of saturation at a material point within the composite idealized as an equivalent homogenous medium; $\bar{D}$ the diffusion coefficient at room temperature, $t$ the 
time coordinate; and, $z$ the depth coordinate. Equation 1 assumes a one-dimensional moisture ingress process through the thickness direction of the composite since moisture exposure from one side of the specimen is considered in this study. An approximate solution to Eq. 1 is:

$$
\bar{s}(z, t)=\frac{4}{z} \sqrt{\frac{\bar{D} t}{\pi}}
$$

Moisture absorption process in vinyl-ester and epoxy matrix composites exhibit deviations from the Fickian model. Glass fiber reinforced polymer composites (GFRP) [10] and carbon fiber reinforced polymer composites (CFRP) with different types of matrices have shown deviations from classical Fickian response which points to additional moisture absorption mechanisms (e.g., reaction processes). For instance, Karbhari and Zhang [2] report a two-stage diffusion process in EVE composite. They present a moisture absorption curve which shows an initial increase followed by a plateau, and another increase in mass of absorbed water as a function of moisture exposure duration. Tsotsis and Weitsman [11] present a simple graphical solution to calculate the diffusion parameters from a non-Fickian moisture absorption response for composites. Despite such deviations, the Fickian model remains to represent the process of moisture ingress in various types of composite systems including woven aramid-glass fiber/epoxy [3], glass/vinyl-ester, carbon/vinyl-ester, carbon/epoxy, glass/epoxy composites $[6,12]$. In addition to composite systems, the response of neat vinyl-ester resin has also been assumed to be Fickian in nature by Sagi-Mana et al. [13].

Murthy et al. [12] provides the coefficient of diffusion at room temperature, $\bar{D}$, for an identical construction of EVE composite as $2.1153 \times 10^{-7} \mathrm{~mm}^{2} / \mathrm{s}$ based on experimental observations. Figure 2 illustrates the through-thickness saturation profiles of specimens for various exposure durations, computed using Eq. 2. The time required for complete saturation for the thickness of the EVE composite is calculated as approximately 9 months. The experimental data confirms this value of diffusion coefficient as inferred from CAI strength values that do not change significantly after the predicted saturation time of 9 months as shown in Table. 1. 


\section{Modeling CAI Failure of Seawater-Exposed Composites}

The geometry and discretization employed to idealize the CAI specimens are illustrated in Fig. 3. The specimens are modeled as a $101.6 \mathrm{~mm} \times 152.4 \mathrm{~mm}$ rectangular plates with a thickness of $5 \mathrm{~mm}$. The displacement in the thickness direction is constrained $\left(\mathrm{u}_{3}=0\right)$ along the edges of the front and back side of the plate. The bottom edge is constrained $\left(u_{2}=0\right)$ and vertical displacement is prescribed at the top surface. CAI response of the composite specimen is modeled by considering the following failure mechanisms:(1) the effect of seawater on constituent stiffness; (2) the effect of seawater on constituent strength; (3) impact induced damage; (4) progressive damage in seawater-exposed composite plies; and, (5) progressive delamination along ply interfaces.

\subsection{Effect of seawater on constituent stiffness}

Moisture ingress affects the stiffness of the vinyl-ester matrix through two competing mechanisms [14]: plasticization due to water sorption that tends to decrease the stiffness accompanied by embrittlement of the matrix material, and leaching of the low molecular weight substances during the hydrolysis process [15] that tends to increase the stiffness of the matrix material. Apicella et al. [16] reported a $10 \%$ increase in the elastic modulus of vinyl-ester at a water saturation level of $45 \%$, which indicates that the leaching process overcomes the plasticization effect up to the reported saturation levels. Experimental reports on vinyl-ester stiffness for higher saturation values is not available in the literature to the best of the authors' knowledge. In this study, the variation of elastic modulus of the vinyl-ester matrix with saturation percentage is assumed to be according to the following expression:

$$
E^{(m)}(\bar{s})=E_{\mathrm{dry}}^{(m)}+\left(E_{\mathrm{sat}}^{(m)}-E_{\mathrm{dry}}^{(m)}\right)\left(1-\exp \left(\frac{-k \bar{s}}{1-\bar{s}}\right)\right)
$$

where, $E^{(m)}(\bar{s})$ and $E_{\text {dry }}^{(m)}$ denote the elastic modulus of the matrix at saturation percentage of $\bar{s}$ and at dry state, respectively; $\mathrm{k}$ is expressed as

$$
k=\left(1-\frac{1}{s_{p s}}\right) \log \left(\frac{E_{\mathrm{sat}}^{(m)}-E_{\mathrm{ps}}^{(m)}}{E_{\mathrm{sat}}^{(m)}-E_{\mathrm{dry}}^{(m)}}\right)
$$


in which, $E_{p s}$ is the matrix modulus at a prescribed saturation percentage of $s_{p s}$.

The variation of the elastic modulus of the matrix as a function of saturation provided

by the proposed model (using $E_{\mathrm{dry}}^{(m)}=3.4 \mathrm{GPa}, E_{\mathrm{ps}}^{(m)}=3.74 \mathrm{GPa}$, and $\bar{s}_{p s}=0.45$ ) and available experimental data are shown in Fig. 4. The proposed model assumes that at longer times, low molecular weight substances are depleted and relative effect of slower-process of water plasticization increases, leading to tapering off of the elastic modulus amplitude at high levels of saturation.

In the experimenal investigations, the compressive moduli of moisture-exposed CAI composite specimens do not show significant reductions as a function of moisture exposure durations. This points to the observation that the fiber stiffness is not significantly altered by the moisture exposure.

\subsection{Effect of seawater on constituent strength}

Moisture ingress significantly reduces the strength of the vinyl-ester matrix. The degradation of matrix strength is attributed to the formation of voids and cracks within the material during leaching of the low molecular weight substances [15]. Moisture induced voids and cracks are randomly dispersed across the wet region and act as fracture initiation sites when subjected to mechanical loading. Apicella et al. [16] reported $57 \%$ reduction in the failure strain of partially-saturated $(\bar{s}=45 \%)$ vinyl-ester specimens under tensile loading. In this study, tensile strength of the matrix material is assumed to decrease with saturation percentage based on the following exponential form:

$$
\sigma_{f}^{(m)}(\bar{s})=\sigma_{f(\mathrm{dry})}^{(m)} \exp \left[-\frac{\bar{s}}{\bar{s}_{p s}} \ln \left(\frac{\sigma_{f(\mathrm{dry})}^{(m)}}{\sigma_{f(\mathrm{ps})}^{(m)}}\right)\right]
$$

where, $\sigma_{f}^{(m)}(\bar{s})$ is tensile strength as a function of saturation; $\sigma_{f(\mathrm{dry})}^{(m)}$ and $\sigma_{f(\mathrm{ps})}^{(m)}$ are values of the tensile strength at dry state and a prescribed saturation of $\bar{s}_{p s}$, respectively. The variation of vinyl-ester matrix strength as a function of saturation provided by the proposed model (using $\sigma_{f(\mathrm{dry})}^{(m)}=67.8 \mathrm{MPa}, \sigma_{f(\mathrm{ps})}^{(m)}=52.08 \mathrm{MPa}$, and $\bar{s}_{p s}=0.45$ ) is shown in Fig. 4 The existing experimental investigations focus on deterioration of tensile strength of 
vinyl-ester matrix when exposed to moisture. In contrast, no experimental data is available on compressive strength deterioration of moisture-exposed vinyl-ester. Considering leaching-induced randomly distributed crack and void formation during the moisture ingress process, tensile and compressive loading conditions lead to similar stress concentrations within the moisture-exposed regions. In this study, the degradation of the compressive strength of vinyl-ester is taken to be identical to degradation under tensile loading.

The strength of E-glass fibers are known to deteriorate in moisture environments $[5,17,18]$. The deterioration has been attributed to generation of surface flaws due to dissolution [18]. Ramirez et al. [19] provided an experimentally calibrated empirical tensile strength degradation model for E-glass fibers exposed to sea water. The extent of strength degradation in fibers were up to $45 \%$ of dry fiber strength near full saturation. Our numerical investigations reveal that at the state of sublaminate buckling in CAI specimens, the stresses within fibers remain below the degraded failure strength. Therefore, reduction in the fiber strength does not significantly affect the CAI strength in E-glass fiber reinforced vinyl-ester composites.

\subsection{Impact induced damage}

Experiments indicate three distinct damage mechanisms when the specimens are subjected to impact: (1) horizontal and vertical matrix cracks at the impact site of the specimens; (2) delamination within a near-conical zone (with apex at the center of the impact surface and base at the back face along the thickness direction of the specimens; and, (3) matrix and fiber cracking within the delamination zone. In the current study, a $35.2 \mathrm{~mm}$ long horizontal crack with $0.625 \mathrm{~mm}$ depth is introduced in the numerical specimens. Experiments suggest that the vertical matrix crack does not grow upon compressive loading, and is not modeled in our simulations. Within the conical impact-damage zone, the composite plies are taken to be completely debonded and a negligible residual strength is assigned to the plies within the impact-damage zone. The base of the conical zone has a diameter of $35 \mathrm{~mm}$ based on the experimental observations. The experiments show no significant change in the initial impact damage zone as a function of seawater exposure duration. The full details on modeling of the initial impact-induced damage in composite specimens is explained in Ref. [8]. 


\subsection{Multiscale ply failure model}

A moisture-dependent continuum damage mechanics model is proposed to idealize the failure processes within the moisture-exposed composite constituents. The proposed model is implemented within the context of the eigendeformation based reduced order homogenization method (EHM) recently proposed by Oskay and coworkers [20, 21]. In the EHM approach, failure response of the composite constituents (i.e., matrix and fiber) is evaluated numerically at the scale of the representative volume element (RVE) and the overall composite response is computed based on the computational homogenization method by appropriate averaging across the RVE domain. The details of the EHM methodology is described in Refs. [20, 21] and the moisture-dependent failure model for constituent materials is described herein.

The RVE of the woven E-glass fiber reinforced vinyl-ester composite is shown in Fig. 3c. The RVE consists of the vinyl-ester matrix, as well as the fibers in the warp and fill directions. We consider a damage potential function, $f$, taken to be piecewise constant within the RVE:

$$
f^{(\eta)}\left(\nu^{(\eta)}, r^{(\eta)}\right)=\phi\left(\nu^{(\eta)}\right)-\phi\left(r^{(\eta)}\right) \leq 0
$$

where, $\nu^{(\eta)}$ is the phase damage equivalent strain; $r^{(\eta)}$ the damage hardening variable; and, $\eta=m, w$ or $f$ denotes matrix, warp or fill fibers, respectively. The evolution equations for constituent damage and hardening variables are given as:

$$
\dot{\omega}^{(\eta)}=\lambda \frac{\partial \phi}{\partial \nu^{(\eta)}} ; \quad \dot{r}^{(\eta)}=\lambda
$$

in which, the consistency parameter, $\lambda$, is governed by the Kuhn-Tucker consistency conditions:

$$
\lambda \geq 0 ; \quad f \leq 0 ; \quad \lambda f=0
$$

Kuhn-Tucker conditions ensure that the growth of damage vanishes upon unloading, which is a necessary condition for thermodynamic consistency. Damage process (i.e., $\lambda>0$ ) occurs only when $f=0$. 
Phase damage equivalent strain, $\nu^{(\gamma)}$, is defined as:

$$
\nu^{(\eta)}(\mathbf{x}, t)=\sqrt{\frac{1}{2}\left(\mathbf{F}^{(\eta)} \hat{\boldsymbol{\epsilon}}^{(\eta)}\right)^{T} \hat{\mathbf{L}}^{(\eta)}\left(\mathbf{F}^{(\eta)} \hat{\boldsymbol{\epsilon}}^{(\eta)}\right)}
$$

in which, $\hat{\boldsymbol{\epsilon}}^{(\eta)}$ is the phase average principal strain tensor within constituent phase, $\eta ; \hat{\mathbf{L}}^{(\eta)}$ the tensor of elastic moduli rotated to the principal directions of the phase-average strain, and; $\mathbf{F}^{(\eta)}$ the weighting matrix, which accounts for the anisotropy in the damage accumulation under tensile and compressive loading:

$$
\begin{gathered}
\mathbf{F}^{(\eta)}(\mathbf{x}, t)=\operatorname{diag}\left(h_{1}^{(\eta)}, h_{2}^{(\eta)}, h_{3}^{(\eta)}\right) \\
h_{\xi}^{(\eta)}(\mathbf{x}, t)=\frac{1}{2}+\frac{1}{\pi} \arctan \left(c_{1}^{(\eta)} \hat{\epsilon}_{\xi}^{(\eta)}\right) ; \quad \zeta=1,2,3
\end{gathered}
$$

where, $\operatorname{diag}(\cdot)$ denotes diagonal matrix; $\epsilon_{\xi}^{(\eta)}$ the components of $\hat{\boldsymbol{\epsilon}}^{(\eta)} ;$ and, $c^{(\eta)}$ a material constant parameterizing the tensile-compressive anisotropy.

The moisture-dependent phase damage evolution function, $\theta$, is expressed based on an arctangent law of the form:

$$
\Phi^{(\eta)}=\frac{\arctan \left[a^{(\eta)} \nu^{(\eta)}-b^{(\eta)}(\bar{s})\right]+\arctan \left[b^{(\eta)}(\bar{s})\right]}{\pi / 2+\arctan \left(b^{(\eta)}(\bar{s})\right]}
$$

in which, $a^{(\eta)}$ and $b^{(\eta)}$ characterize the shape of the phase-average stress-strain relationship within constituent, $\eta . b^{(\eta)}$ is taken to be a nonlinear function of saturation to account for the reduction of strength as a function of moisture within the material:

$$
b^{(\eta)}=b_{0}^{(\eta)}+b_{1}^{(\eta)} \bar{s}+b_{2}^{(\eta)} \bar{s}^{2}
$$

where, $b_{0}^{(\eta)}, b_{1}^{(\eta)}$, and $b_{2}^{(\eta)}$ are material parameters.

The proposed saturation-dependent multiscale ply failure model contains a total of nine parameters to describe failure in the matrix phase (i.e., $a^{(m)}, b_{0}^{(m)}, b_{1}^{(m)}, b_{2}^{(m)}$ and $\left.c^{(m)}\right)$; as well as the warp- (i.e., $a^{(w)}, b_{0}^{(w)}$ and $\left.c^{(w)}\right)$ and fill-direction $\left(a^{(f)}, b_{0}^{(f)}\right.$ and $\left.c^{(f)}\right)$ fiber phases. The failure parameters of the composite constituents at dry state are calibrated against experimental data and observations that are provided in Ref. [22]. The calibrations 
are conducted by minimizing the discrepancy between the RVE-scale simulations and experimentally observed composite strength under compressive and tensile loading. The calibrated dry material parameters for the composite constituents are summarized in Table 2. The overall composite strength computed using the calibrated material parameters are: 518.6 $\mathrm{MPa}$ and $353.4 \mathrm{MPa}$ along the warp and fill-directions, respectively, under tensile loading, and 358.2 $\mathrm{MPa}$ and $338.7 \mathrm{MPa}$ along the warp and fill-directions, respectively, under compressive loading. The simulated composite strength properties are in close agreement with experimental observations of $512.5 \mathrm{MPa}$ and $350.9 \mathrm{MPa}$ along the warp and fill-directions, respectively, under tensile loading, and 363.4 MPa and 336.2 MPa along the warp and fill-directions, respectively, under compressive loading.

$b_{1}^{(m)}$ and $b_{2}^{(m)}$, which characterize the effect of moisture on failure characteristics of vinyl-ester resin, are calibrated based on the strength degradation model provided by Eq. 5 and the experimental data provided by Apicella et al. [23]. The calibrated material parameters are $b_{1}^{(m)}=-148.68$ and $b_{2}^{(m)}=45.21$, respectively. Figure 6 illustrates the stress-strain response of the composite when subjected to tensile and compressive loading along the warp and fill directions, as well as the effect of the moisture-ingress on the early constitutive response due to accelerated matrix cracking due to moisture absorption.

\subsection{Interlaminar failure model}

Progressive delamination of the plies upon compressive loading is idealized using a saturation-dependent cohesive surface model, which provides the traction-displacement relationship along the interlaminar boundaries as illustrated in Fig. 7:

$$
\mathbf{t}=\left[1-\omega_{\text {int }}(\bar{s})\right] \mathbf{K}(\bar{s}) \boldsymbol{\delta}
$$

where, $\mathbf{t}$ denotes traction vector along the ply interfaces; $\mathbf{K}$ the saturation dependent tensor of interface stiffnesses; $\boldsymbol{\delta}$, the displacement jump across ply interfaces; and, $\omega_{\text {int }}$ denotes the interlaminar damage variable, whose evolution is expressed as [8]:

$$
\dot{\omega}_{\text {int }}=\left\{\begin{array}{ccc}
\frac{\kappa_{f}(\bar{s}) \kappa_{c}}{\kappa^{2}\left(\kappa_{f}(\bar{s})-\kappa_{c}\right)} \dot{\kappa} & \text { if } & \lambda_{c}=1 \\
0 & \text { if } & \lambda_{c}<1
\end{array}\right.
$$


in which, $\lambda_{c}=\sqrt{\left(\left\langle\delta_{N}\right\rangle / \delta_{N}^{c}\right)^{2}+\left(\left\|\boldsymbol{\delta}_{S}\right\| / \delta_{S}^{c}\right)^{2}}$ is damage initiation criteria; $\delta_{N}$ and $\boldsymbol{\delta}_{S}$ the normal and tangential components of the displacement jump vector; $\delta_{N}^{c}$ and $\delta_{S}^{c}$ the material parameters describing the initiation of interface damage; $\kappa_{c}=\sqrt{\left(\delta_{N}^{c}\right)^{2}+\left(\delta_{S}^{c}\right)^{2}}$ denotes critical equivalent displacement jump at damage initiation; $\kappa$ the maximum equivalent displacement jump throughout the loading history; and,

$$
\kappa_{f}(\bar{s})=\sqrt{\frac{4 G_{c}^{2}(\bar{s})}{\left(t_{N}^{f}(\bar{s})\right)^{2}+\left(t_{S}^{f}(\bar{s})\right)^{2}}}
$$

where, $t_{N}^{f}$ and $t_{S}^{f}$ are ultimate normal and shear tractions along the ply interface, respectively; and, $G_{c}$ critical energy release rate modeled using the Benzeggah-Kenane criterion [24] for mixed mode conditions.

The calibration of the ply interface parameters for dry CAI specimens are described in Ref. [8]. In this manuscript, we concentrate on the effect of moisture on the interface delamination response characteristics. The interface stiffness coefficients in the normal and tangential directions, $K_{N}$ and $K_{S}$, respectively, are taken to be based on the homogenized moduli of the composite material along the thickness direction (see Fig. 3): $K_{N}=\bar{E}_{33} / h$ and $K_{S}=\bar{G}_{23} / h$ ( $h$ is the interface region thickness taken to be $\left.100 \mu \mathrm{m}\right)$. The homogenized moduli of the composite are saturation dependent due to saturation-dependent matrix stiffness, modeled by Eq. 4. The ultimate normal and shear tractions along the interface are taken to be equal to the saturation-dependent normal and shear strength of the composite along the thickness direction: $t_{N}^{f}=\bar{\sigma}_{33}^{f}$ and $t_{S}^{f}=\bar{\tau}_{23}^{f}$. The thickness direction normal and shear strength of the composite degrade as a function of saturation due to the saturation-dependent matrix strength, modeled by Eq. 5. The critical energy release rate at a given saturation level is computed by assuming that the displacement jump at failure remains unaltered in moist and dry specimens as illustrated in Fig. 7. In case of unsaturated specimens, the interface strength between each ply is different and is calculated using the ratio of the unsaturated over the saturated calibrated strength of the RVE of the woven EVE composite in the $\mathrm{z}$ direction. 


\section{$5 \quad$ Numerical Simulation of Compression Failure in Seawater-Exposed CAI Specimens}

We conducted a series of numerical simulations to predict the compression failure of EVE composite specimens subjected to moisture exposure and impact loading. Compression failure of CAI specimens that are exposed to seawater environment for durations of $0,1,2,3$, $5,8,10$, and 30 months are considered. The moisture state through the thickness of each specimen is idealized using a piecewise constant approximation, where, saturation in each ply is taken to be a constant value, equal to the average saturation computed using Eq. 2 (see Fig. 2). Ply saturation values for each specimen employed in our numerical simulations summarized in Table 3.

The compression simulations consider the failure mechanisms of impact-induced damage, progressive failure within the composite constituents, as well as progressive delamination along the ply interfaces. Experiments indicate that the impact-induced damage profiles for partially- and fully-saturated specimens do not significantly differ from the damage profile for the dry specimen [7]. Identical impact-induced damage profiles are considered for the dry and all moisture-exposed specimens.

\section{$5.1 \quad$ Normalized CAI strength}

The experimentally observed CAI strength of composite specimens as a function of seawater-exposure is shown in Fig. 8a. The measured CAI strength of the composite specimens subjected to 4 months of seawater exposure is $5.6 \%$ higher compared to the dry CAI strength, whereas the CAI strength of saturated specimens (i.e., seawater exposure duration of 9 months and more) is smaller compared to dry CAI strength. The relative increase in the CAI strength in unsaturated composite specimens is attributed to the higher specimen thicknesses (shown in Table 1).

The CAI strength of composites is defined by sublaminate buckling, which is a function of the specimen thickness. Considering a classical Euler buckling formula for a pin supported 
column, The buckling stress, $\sigma_{\mathrm{cr}}$ is expressed as:

$$
\sigma_{\mathrm{cr}}=\frac{\pi^{2} E}{(\mathrm{E} / r)^{2}}
$$

where, $E$ the modulus of elasticity; $L$ the unsupported length of the column; $r=\sqrt{I / A}$ the smallest radius of gyration of the column; $I$ the moment of inertia; and, $A$ the cross-sectional area. For a rectangular cross-section $\left(I=b t^{3} / 12\right.$ and $A=b t$, with $b$ and $t$ the column width and thickness, respectively), the buckling stress is proportional to the square of the column thickness. We describe the CAI response of composite specimens based on a normalized CAI strength parameter:

$$
s_{C A I}=\frac{\sigma_{C A I}}{t^{2}} \cdot \frac{\left(\mathcal{L}^{\prime}\right)^{2}}{\bar{E}_{c}}
$$

in which, $\sigma_{C A I}$ is the observed CAI strength of specimen with thickness t; $\mathcal{L}^{\prime}$ the sublaminate length prior to buckling; and, $\bar{E}_{c}$ the compression modulus of the composite.

Figure 8 illustrates the variation of $s_{C A I}$ as a function of duration of moisture exposure observed in the experiments, as well as those predicted by the numerical simulations. For all specimens, $\bar{E}_{c}=31.2 \mathrm{GPa}$ and $\mathcal{L}^{\prime}=36 \mathrm{~mm}$.

The variation of $s_{C A I}$ with moisture exposure duration predicted by the numerical simulations captures the trend that moisture exposure leads to a reduction in the normalized CAI strength of moisture exposed specimens compared to dry composites. The simulations reveal that there is a significant reduction in $s_{C A I}$ upon a slight exposure to moisture, which is partially recovered upon full saturation. The overall reduction of the normalized CAI strength (from dry conditions) for the saturated composites predicted by the numerical simulations are $15.5 \%$ compared to $23 \%$ reduction in unsaturated specimens. We attribute higher reduction in $s_{C A I}$ in unsaturated specimens compared to saturated composites to the uneven material properties in the unsaturated specimens with more degradation along the moisture exposure face, which promotes buckling. While the current experiments seem to agree with this trend, further independent CAI experimentation on unsaturated specimens is needed to validate the numerical predictions and the underlying reason for the observed behavior. While, the overall trend of the CAI strength is successfully modeled by the 
proposed multiscale CAI failure model, the simulations predict a somewhat lower CAI strength of dry and moisture-exposed specimens compared to the experimental observations. The discrepancy between simulated and experimentally observed CAI strength is due to the uncertainties in the impact-induced damage within the composite constituents and ply interfaces, as well as in the characterization of the moisture-induced material property changes due to moisture ingress. Additional experimental and numerical investigations to more accurately identify damage processes at the micro- and specimen scale are currently on-going.

\section{Conclusion}

In this manuscript, we presented a numerical investigation of the effect of moisture ingress on the compression-after-impact response of E-glass vinyl-ester composite materials. A moisture dependent multiscale composite damage model is proposed and employed to predict the variation of CAI strength as a function of moisture exposure durations. One key observation is that partially-saturated specimens display lower CAI strength compared to fully-saturated specimens. This observation is in agreement with experiments. The proposed multiscale damage model was shown to be effective in capturing moisture exposure induced degradation

of material as well as CAI parameters. Additional difficulties related to modeling the effect of seawater exposure on CAI remains to be investigated. First, more experimental investigation is needed in the partially-saturated range to further validate the prediction of the model. Second, incorporation of the effects of seawater exposure on matrix-fiber debonding as well as on delamination characteristics are likely to provide a more accurate model for prediction of CAI response. These issues will be addressed in future studies.

\section{Acknowledgements}

The authors acknowledge the financial support from the Office of Naval Research and Vanderbilt University. The authors would further like to thank Prof. Roy Xu at University of Texas, El Paso for fruitful discussions and experimental collaboration. 


\section{References}

[1] A.P. Mouritz and Z. Mathys. Post-fire mechanical properties of marine polymer composites. Compos. Struct., 47(1-4):643-653, 1999.

[2] V.M. Karbhari and S. Zhang. E-glass/vinylester composites in aqueous environments-I: experimental results. Appl. Comp. Mater., 10(1):19-48, 2003.

[3] K. Imieliska and L. Guillaumat. The effect of water immersion ageing on low-velocity impact behaviour of woven aramid-glass fibre/epoxy composites. Compos. Sci. Technol., 64(13-14):2271-2278, 2004.

[4] L.V. Smith and Y.J. Weitsman. Sea water effects on the fatigue response of polymeric composites. Progress in Durability Analysis of Composite Systems(USA), pages 217-223, 1996.

[5] K. Liao, C.R. Schultheisz, and D.L. Hunston. Effects of environmental aging on the properties of pultruded GFRP. Composites B, 30(5):485-493, 1999.

[6] R. Roy, B.K. Sarkar, and N.R. Bose. Effects of moisture on the mechanical properties of glass fibre reinforced vinylester resin composites. Bull. Mater. Sci., 24(1):87-94, 2001.

[7] L.R. Xu, A. Krishnan, H. Ning, and U. Vaidya. A novel approach to evaluate dynamic failure and durability of woven e-glass/vinyl ester composite. In review.

[8] H. Yan, C. Oskay, A. Krishnan, and L.R. Xu. Compression After Impact Response of Woven Fiber-Reinforced Composites. Compos. Sci. Technol., 70(14):2128-2136, 2010.

[9] S. Pillay, U.K. Vaidya, and G.M. Janowski. Liquid molding of carbon fabric-reinforced nylon matrix composite laminates. J. Thermoplast. Compos. Mater., 18(6):509, 2005.

[10] E.P. Gellert and D.M. Turley. Seawater immersion ageing of glass-fibre reinforced polymer laminates for marine applications. Composites A, 30(11):1259-1265, 1999.

[11] T.K. Tsotsis and Y. Weitsman. A simple graphical method for determining diffusion parameters for two-stage sorption in composites. J. Mater. Sci. Lett., 13(22):1635-1636, 1994. 
[12] H.N. Narasimha Murthy, M. Sreejith, M. Krishna, S.C. Sharma, and T.S. Sheshadri. Seawater Durability of Epoxy/Vinyl Ester Reinforced with Glass/Carbon Composites. J. Reinf. Plast. Compos., 29(10):1491, 2010.

[13] D. Sagi-Mana, M. Narkis, A. Siegmann, R. Joseph, and H. Dodiuk. The effect of marine environment on a vinyl ester resin and its highly filled particulate quartz composites. $J$. Appl. Polym. Sci., 69(11):2229-2234, 1998.

[14] A. Apicella, C. Migliaresi, L. Nicodemo, L. Nicolais, L. Iaccarino, and S. Roccotelli. Water sorption and mechanical properties of a glass-reinforced polyester resin. Composites, 13(4):406-410, 1982.

[15] MK Antoon and JL Koenig. Irreversible effects of moisture on the epoxy matrix in glass-reinforced composites. J. Polym. Sci., 19(2):197-212, 1981.

[16] A. Apicella and L. Nicolais. Effect of water on the properties of epoxy matrix and composite. Epoxy Resins Compos. I, pages 69-77, 1985.

[17] A.G. Metcalfe and G.K. Schmitz. Mechanism of stress corrosion in E-glass filaments. Glass Technol., 13(1):5-16, 1972.

[18] M.J. Matthewson. Models for fiber reliability. In Proceedings of SPIE, volume 1973, pages 128-137, 1993.

[19] F.A. Ramirez, L.A. Carlsson, and B.A. Acha. Evaluation of water degradation of vinylester and epoxy matrix composites by single fiber and composite tests. J. Mater. Sci., 43(15):5230-5242, 2008.

[20] R. Crouch and C. Oskay. Symmetric meso-mechanical model for failure analysis of heterogeneous materials. Int. J. Mult. Comp. Eng., 8:447:461, 2010.

[21] C. Oskay and J. Fish. Eigendeformation-based reduced order homogenization for failure analysis of heterogeneous materials. Comp. Meth. Appl. Mech. Engng., 196(7):1216-1243, 2007.

[22] S.A. Tekalur, K. Shivakumar, and A. Shukla. Mechanical behavior and damage evolution in E-glass vinyl ester and carbon composites subjected to static and blast loads. Composites B, 39(1):57-65, 2008. 
[23] A. Apicella, C. Migliaresi, L. Nicolais, L. Iaccarino, and S. Roccotelli. The water ageing of unsaturated polyester-based composites: influence of resin chemical structure.

Composites, 14(4):387-392, 1983.

[24] M. L. Benzeggagh and M. Kenane. Measurement of mixed-mode delamination fracture toughness of unidirectional glass/epoxy composites with mixed-mode bending apparatus. Compos. Sci. Technol., 56:439-449, 1996. 


\section{Figure Captions}

Figure 1: (a) Realistic exposure of CAI specimens to seawater; (b) Drop tower impact testing of EVE composite; (c) Compression-after-impact testing of EVE composite; (d) Damage profile after impact testing; (e) Damage profile after CAI testing; (f) Shear crack along the side of the specimen after CAI testing.

Figure 2: Saturation profiles in the composite specimens subjected to seawater exposure.

Figure 3: (a) Composite specimens exposed to seawater on one side only; (b) Geometry and boundary conditions; (c) Representative volume element with matrix (transparent), fill fibers (lateral) and warp fibers (vertical).

Figure 4: Variation of elastic modulus with saturation percentage as predicted by the model.

Figure 5: Stress-strain curves of dry and saturated vinyl-ester matrix subjected to uniaxial compressive and tensile loading.

Figure 6: Stress-strain diagram showing tension and compression properties of dry and saturated (inset) vinyl-ester matrix.

Figure 7: Interface damage evolution model for dry (solid lines) and saturated (dashed lines) composites.

Figure 8: (a) Variation of CAI strength as a function of seawater exposure duration for experimental dry and wet specimens. (b) Variation of normalized CAI strength as a function of seawater exposure duration for experimental and simulated specimens. 


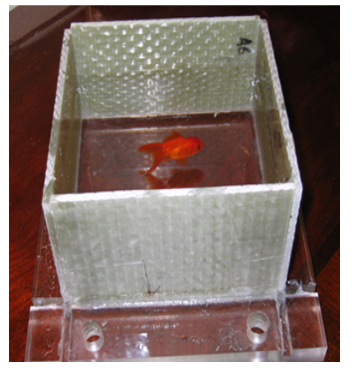

(a)

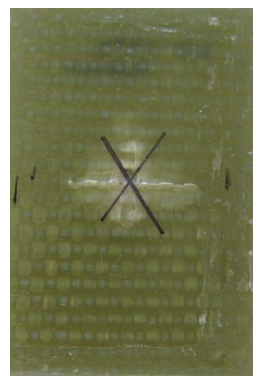

(d)

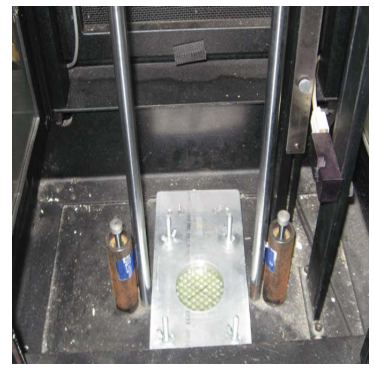

(b)

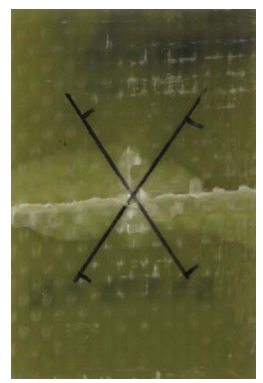

(e)

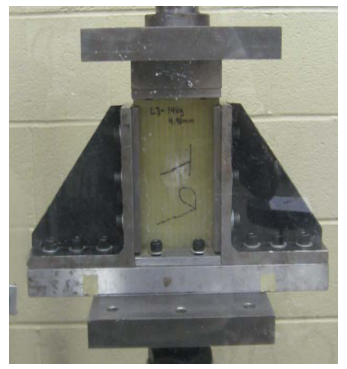

(c)

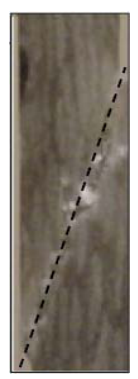

(f)

Figure 1: 


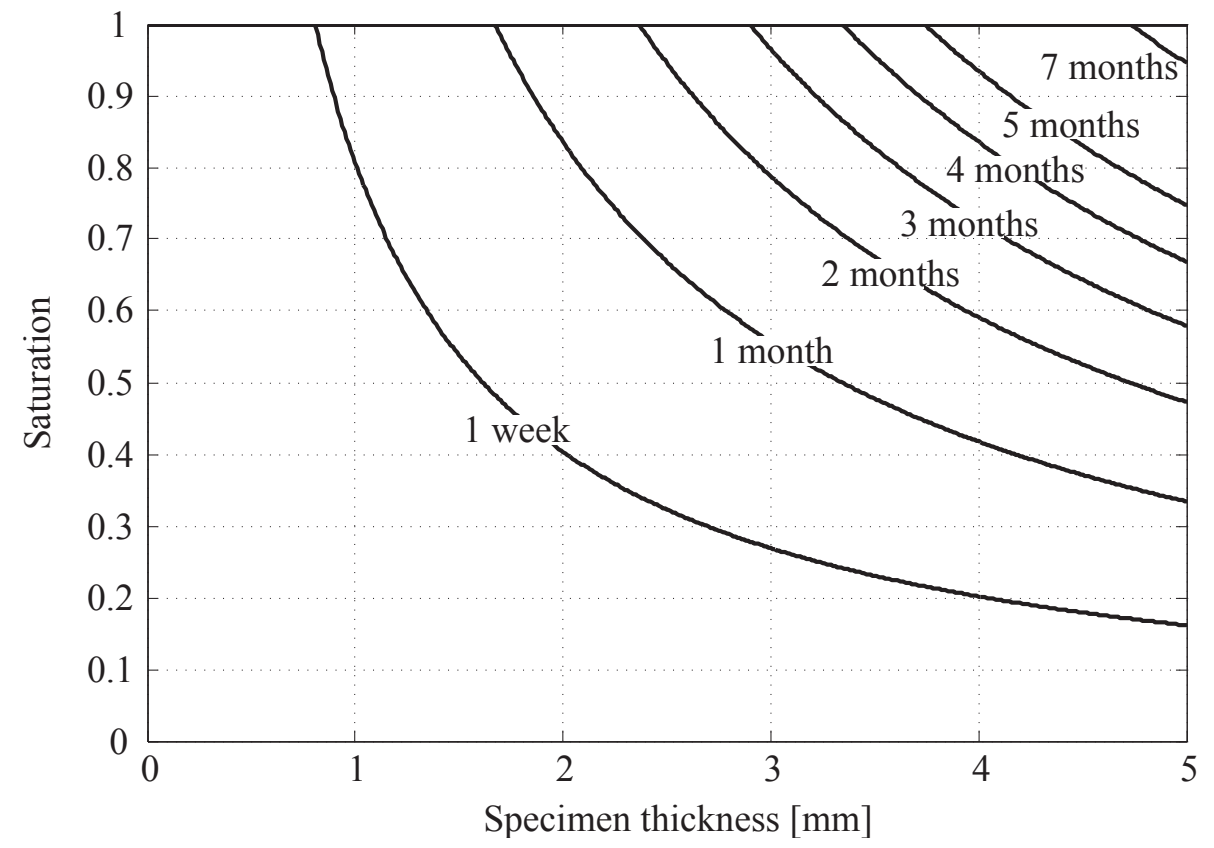

Figure 2: 


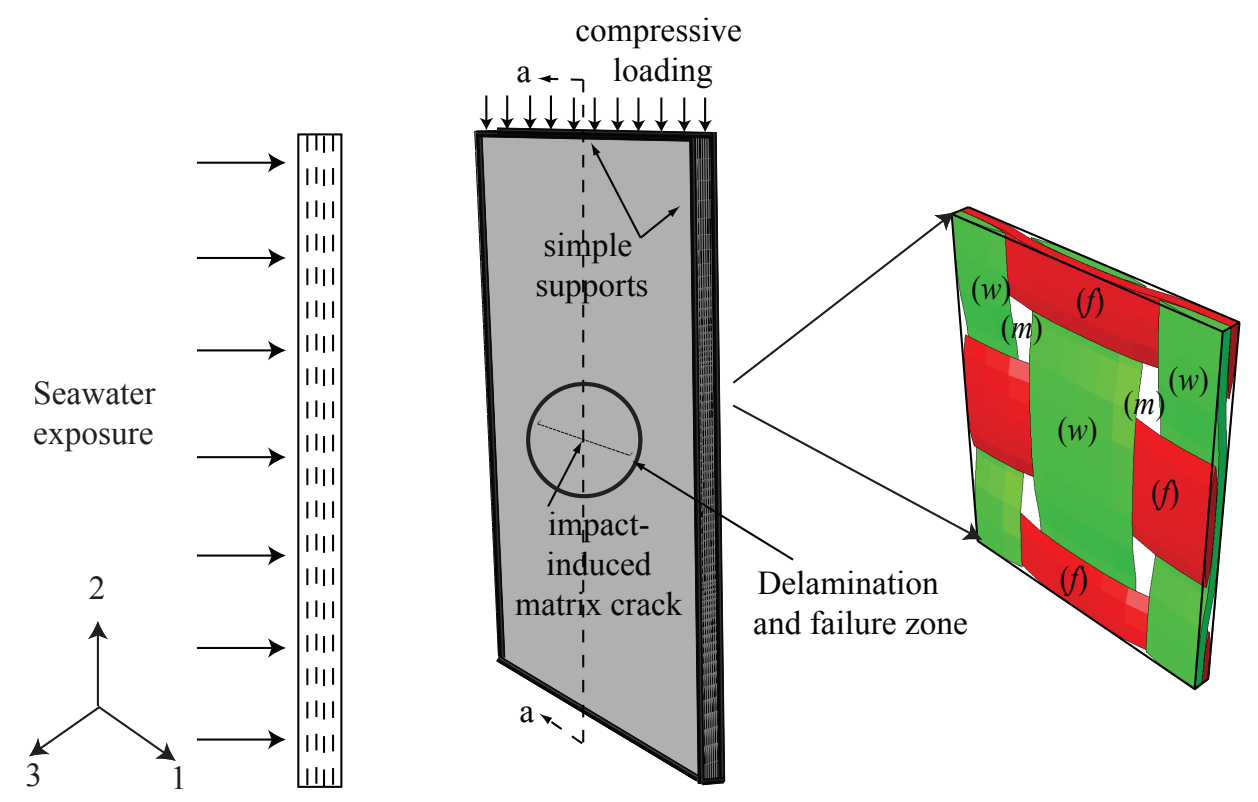

(a)

(b)

(c)

Figure 3: 


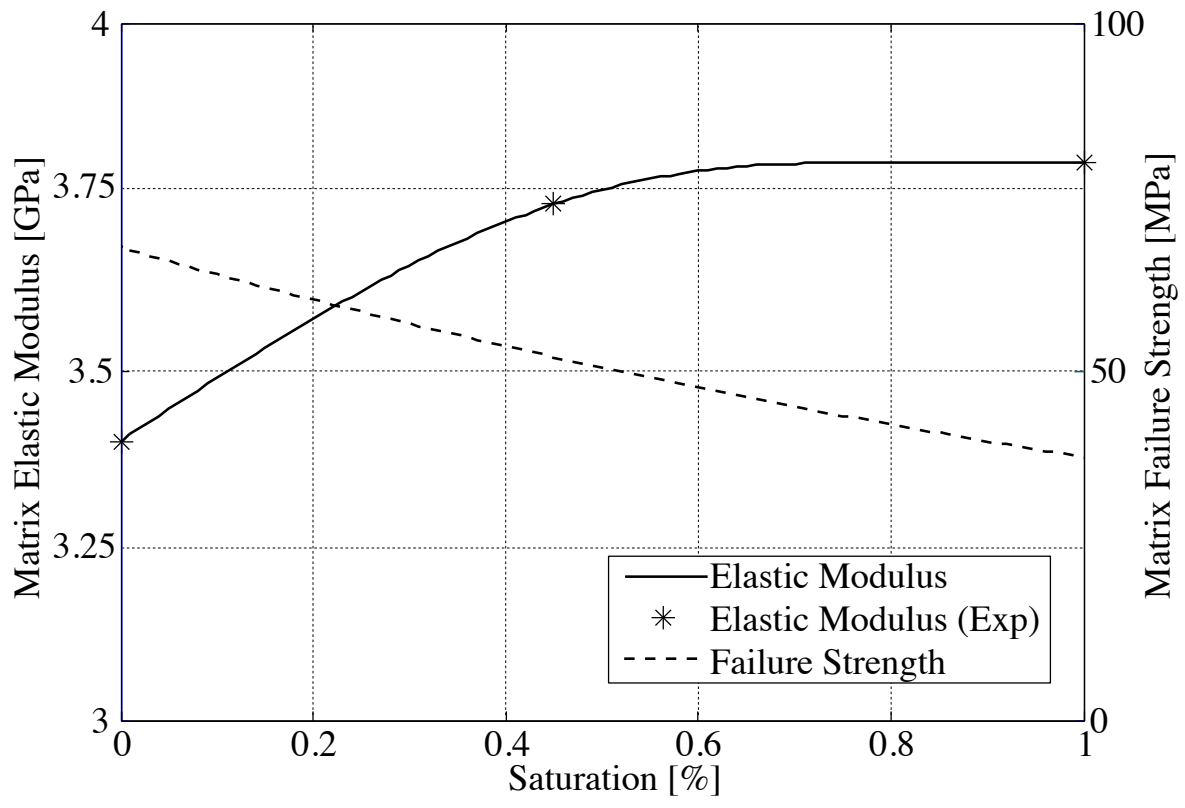

Figure 4: 


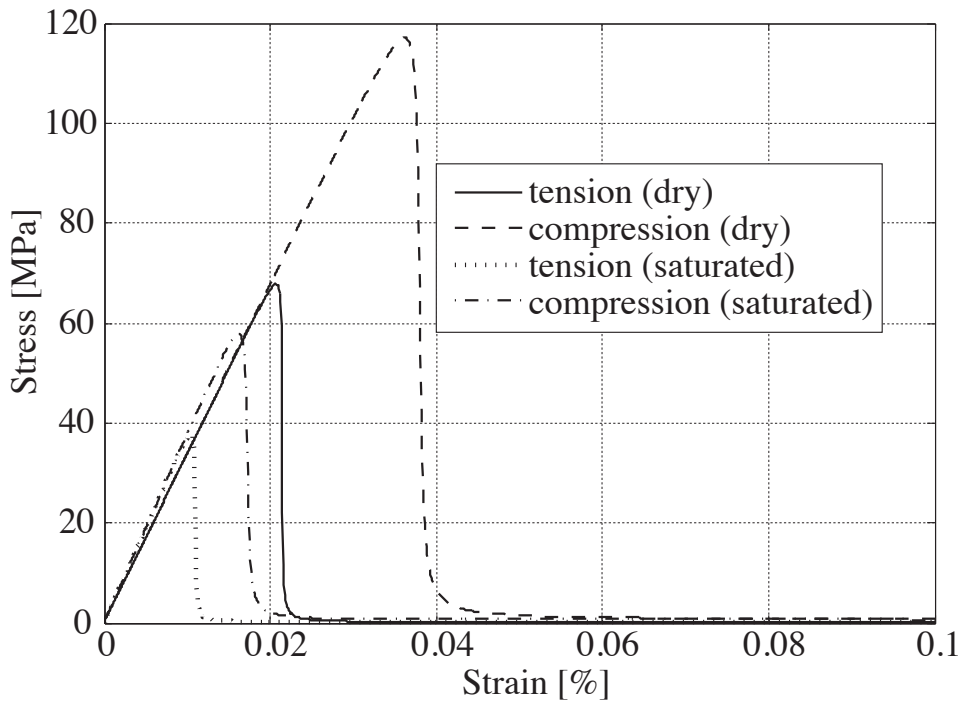

Figure 5: 


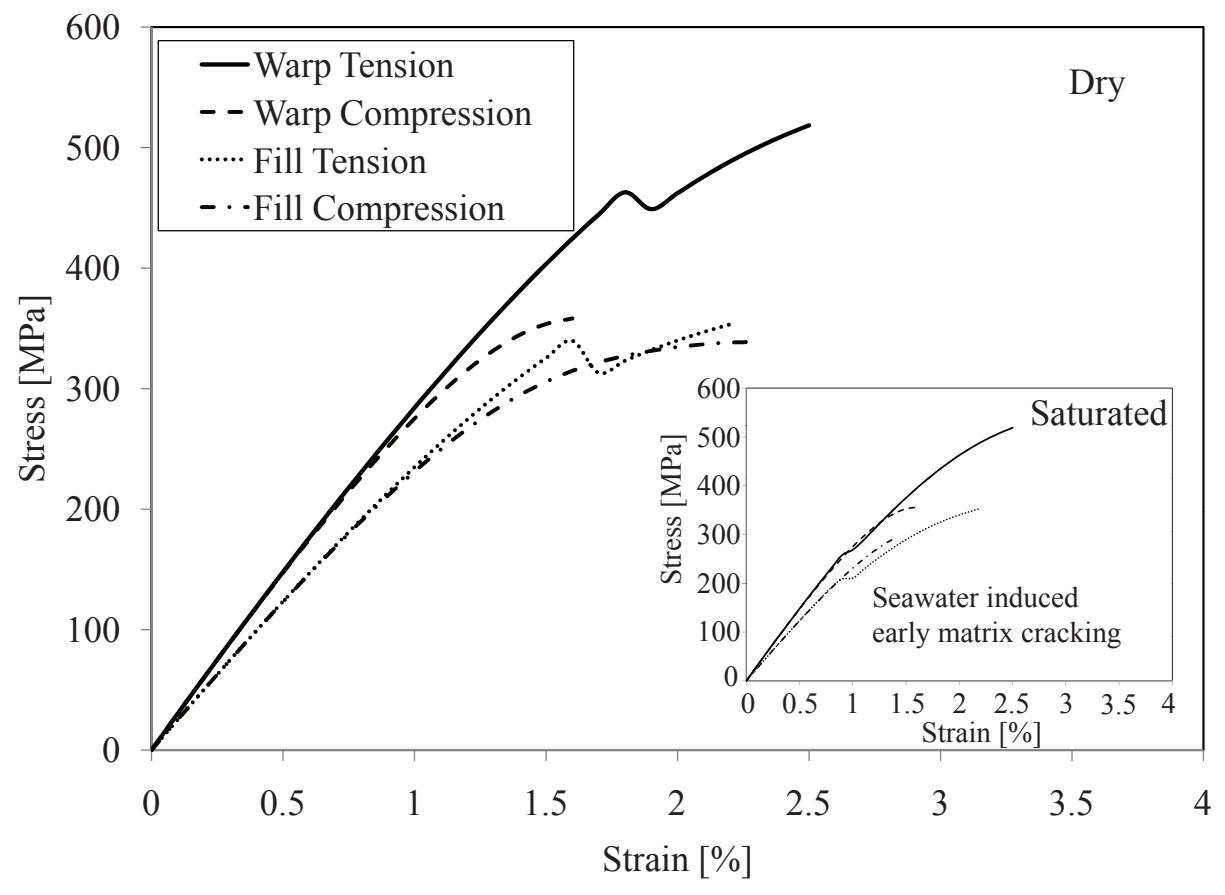

Figure 6: 


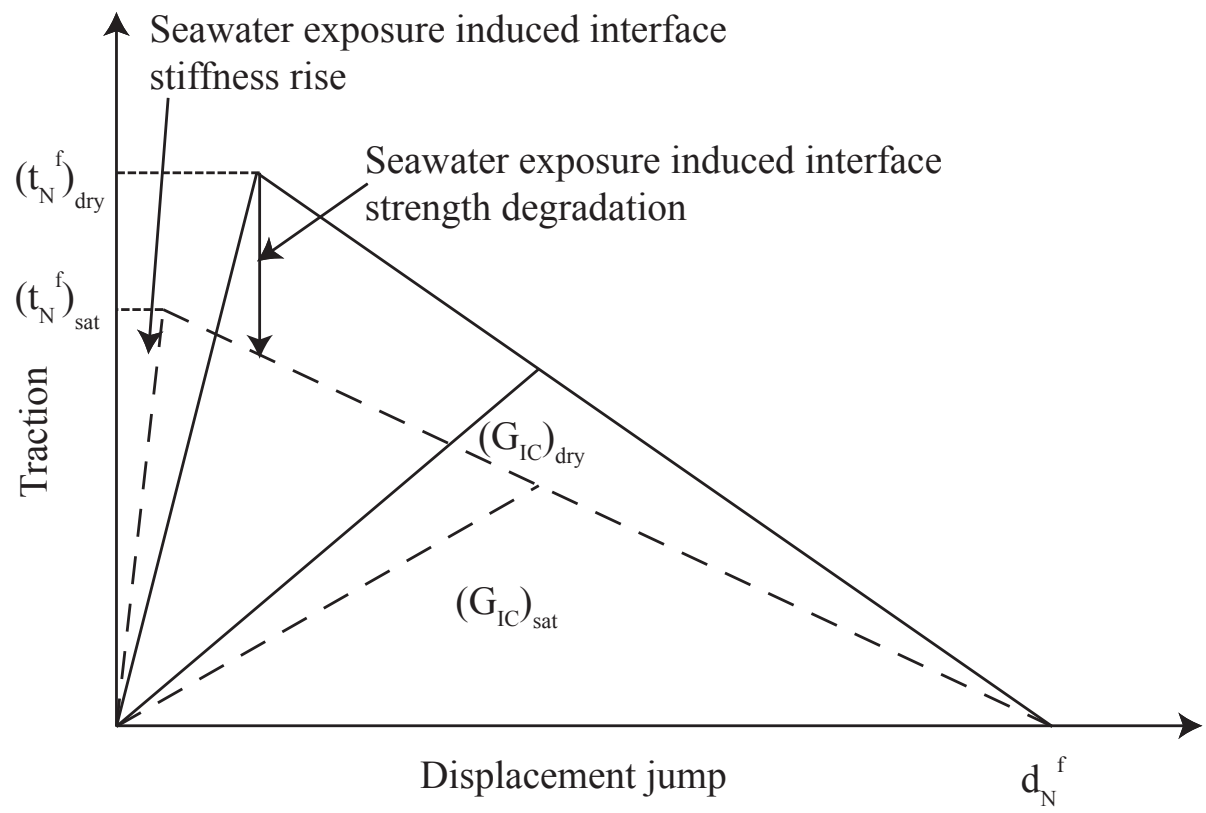

Figure 7: 


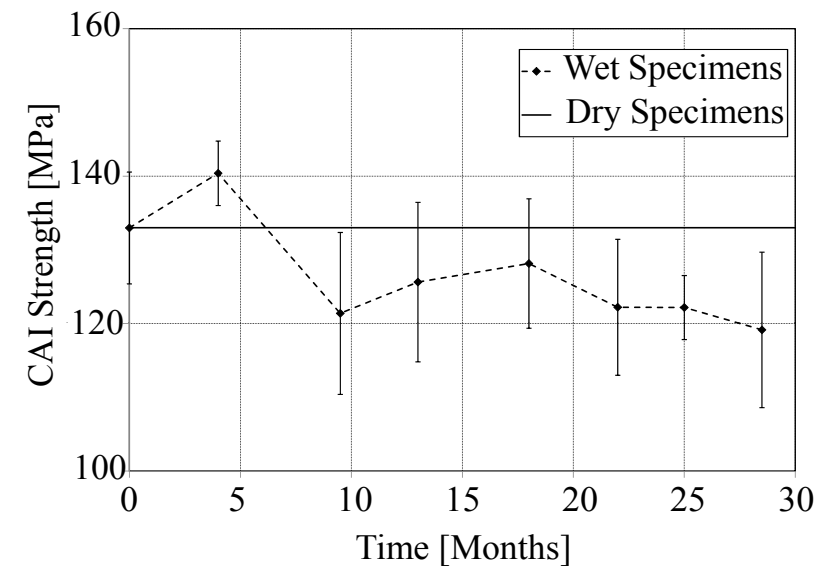

(a)

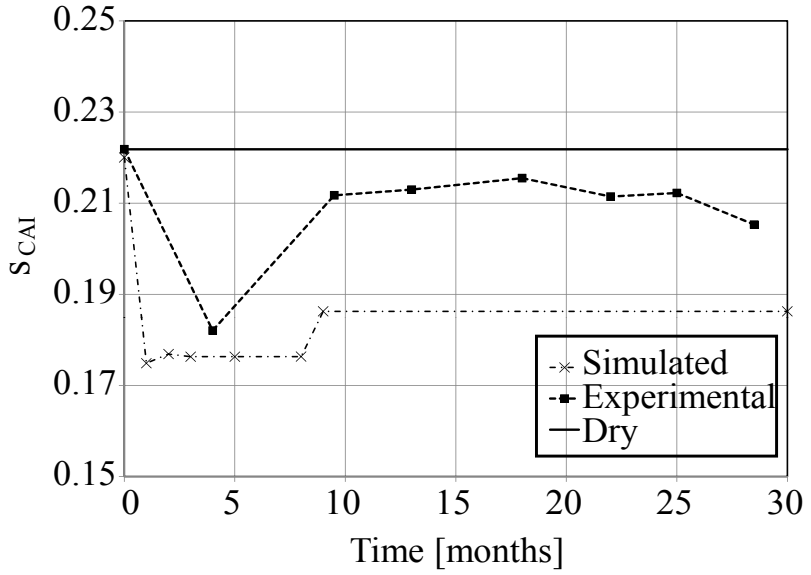

(b)

Figure 8: 
Table 1: Variation of Compression-After-Impact (CAI) Strength with seawater exposure duration.

\begin{tabular}{|c|c|c|c|}
\hline $\begin{array}{c}\text { Time } \\
{[\mathrm{mo}]}\end{array}$ & $\begin{array}{c}\text { CAI strength } \\
{[\mathrm{MPa}]}\end{array}$ & $\begin{array}{c}\text { Change from } \\
\text { dry strength }[\mathrm{MPa}]\end{array}$ & $\begin{array}{c}\text { Average } \\
\text { thickness }[\mathrm{mm}]\end{array}$ \\
\hline 0 & $132.98 \pm 7.59$ & 0 & $4.99 \pm 0.45$ \\
\hline 4 & $140.40 \pm 4.37$ & +5.58 & $5.66 \pm 0.1$ \\
\hline 9.5 & $121.39 \pm 10.98$ & -8.72 & $4.88 \pm 0.06$ \\
\hline 13 & $125.63 \pm 9.05$ & -5.53 & $4.95 \pm 0.11$ \\
\hline 18 & $128.15 \pm 8.77$ & -3.63 & $4.97 \pm 0.16$ \\
\hline 22 & $122.22 \pm 9.22$ & -8.09 & $4.90 \pm 0.06$ \\
\hline 25 & $122.18 \pm 4.34$ & -8.12 & $4.89 \pm 0.07$ \\
\hline 28.5 & $119.15 \pm 10.55$ & -10.4 & $4.91 \pm 0.13$ \\
\hline
\end{tabular}


Table 2: Calibrated failure parameters of composite constituents.

\begin{tabular}{|c|c|c|c|c|c|}
\hline Constituent Parameter & $a^{(\cdot)}$ & $b_{0}^{(\cdot)}$ & $b_{1}^{(\cdot)}$ & $b_{2}^{(\cdot)}$ & $c^{(\cdot)}$ \\
\hline \hline matrix $(m)$ & 400.00 & 212.94 & -148.68 & 45.21 & 15.00 \\
\hline fill $(f)$ & 1.50 & 2.30 & 0 & 0 & 5.00 \\
\hline warp $(w)$ & 1.00 & 2.00 & 0 & 0 & -28.00 \\
\hline
\end{tabular}


Table 3: Variation of average ply saturation with time employed in the numerical simulations.

\begin{tabular}{|c|c|c|c|c|c|c|c|c|}
\hline Time $[\mathrm{mo}]$ & Ply \#1 $^{*}$ & Ply \#2 & Ply \#3 & Ply \#4 & Ply \#5 & Ply \#6 & Ply \#7 & Ply \#8 \\
\hline \hline 1 & 100 & 100 & 94.6 & 78 & 60.2 & 49 & 41.4 & 35.8 \\
\hline 2 & 100 & 100 & 100 & 94.5 & 75.6 & 63 & 54 & 47.3 \\
\hline 3 & 100 & 100 & 100 & 100 & 96.3 & 84.9 & 71.7 & 62 \\
\hline 4 & 100 & 100 & 100 & 100 & 100 & 94.6 & 82.8 & 71.6 \\
\hline 5 & 100 & 100 & 100 & 100 & 100 & 99.6 & 85.4 & 74.7 \\
\hline 8 & 100 & 100 & 100 & 100 & 100 & 100 & 100 & 94.5 \\
\hline 10,30 & 100 & 100 & 100 & 100 & 100 & 100 & 100 & 100 \\
\hline
\end{tabular}

* Ply numbering as shown in Fig. 3. 